INPLASY

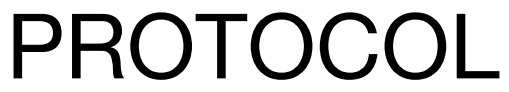

To cite: Dai et al. Gynostemma Pentaphyllum for Dyslipidemia: A protocol of Systematic Review of Randomized Controlled Trials. Inplasy protocol 202210135. doi: 10.37766/inplasy2022.1.0135

Received: 31 January 2022

Published: 31 January 2022

Corresponding author: Jianping Liu

liujp@bucm.edu.cn

Author Affiliation:

Centre for Evidence-Based

Chinese Medicine, Beijing

University of Chinese

Medicine.

Support: Government and university.

Review Stage at time of this submission: Preliminary searches.

Conflicts of interest: None declared.

\section{Gynostemma Pentaphyllum for Dyslipidemia: A protocol of Systematic Review of Randomized Controlled Trials}

Dai, N1; Zhao, FF2; Fang, M33 Kong, LY4; Pu, FL5; Liu, JP6.

Review question / Objective: The aim of this systematic review is to compare Chinese herb Gynostemma pentaphyllum with no treatment, placebo, lipid-lowering agents, or other Chinese herbal products that containing red yeast rice extracts to evaluate effectiveness, safety and costeffectiveness in people with dyslipidemia to inform clinical practice. To this end, the proposed systematic review will address the following question: Is Chinese herb Gynostemma pentaphyllum effective for dyslipidemia?

Information sources: The PubMed, the Cochrane Library, EMBASE, Web of Science, China National Knowledge Infrastructure (CNKI), Chinese Scientific Journal Database (VIP), SinoMed, and Wanfang Data will be searched in January 2022. We also explored the World Health Organization International Clinical Trials Registry Platform (https:// www.who.int/clinical-trials-registry-platform), ClinicalTrials.gov (www.clinicaltrials.gov/), and the Chinese Clinical Trial Registry (http://www.chictr.org.cn/index.aspx) for ongoing or unpublished trials to January 2022. Subject words or text words are determined based on published systematic reviews, clinical practice guidelines, ICD-10, ICD-11, MeSH terms, and Emtree. In addition, the reference lists of all identified studies will also be searched to find any relevant trials for inclusion.

INPLASY registration number: This protocol was registered with the International Platform of Registered Systematic Review and Meta-Analysis Protocols (INPLASY) on 31 January 2022 and was last updated on 31 January 2022 (registration number INPLASY202210135).

\section{INTRODUCTION}

Review question / Objective: The aim of this systematic review is to compare Chinese herb Gynostemma pentaphyllum with no treatment, placebo, lipid-lowering agents, or other Chinese herbal products that containing red yeast rice extracts to evaluate effectiveness, safety and costeffectiveness in people with dyslipidemia to 
inform clinical practice. To this end, the proposed systematic review will address the following question: Is Chinese herb Gynostemma pentaphyllum effective for dyslipidemia?

Rationale: The total glucoside tablets/oral liquid/capsules obtained from Chinese herb gynostemma pentaphyllum were commonly used as lipid-lowering Chinese patent medicines in China from 1990s to early 21st century. However, the use of gynostemma pentaphyllum decreased significantly in recent years due to emerging copeting products such as statins, and other herbal lipid-lowering agents. What is the comparative effectiveness of gynostemma pentaphyllum to other lipid lowering agents?

Condition being studied: Cardiovascular diseases (CVDs) are the leading cause of morbidity and mortality globally. Dyslipidemia is a significant risk factor for the development of CVDs. Therefore, appropriate management of dyslipidemia is essential for the primary prevention of CVDs. Epidemiological data show that the global prevalence of dyslipidemia in 2019 is about $15.2 \%$, and the prevalence of dyslipidemia in adults in some developed countries is as high as $\mathbf{5 5 \%}$. A new large, national cross-sectional study (involving $2,314,538$ participants) found that dyslipidemia is highly prevalent $(33.8 \%)$ in China but commonly undertreated and uncontrolled. Even among people with established atherosclerotic cardiovascular disease (ASCVD) and a high risk of ASCVD, only $26.6 \%$ and $42.9 \%$, respectively, achieved LDL-C control targets. Moreover, statins, the evidence-based lipid-lowering agents recommended by the guidelines, are not available in almost one-half of the primary care institutions, with the lowest available in rural areas. Statins are the most effective agents for improving lipid spectrum, and a minority of patients suffer from side effects that impact treatment adherence and limit the full potential of risk reduction. Some of patients with a proven or perceived intolerance to statins and other established lipid-lowering agents use alternative natural products to control their lipid levels. Some natural herbs, such as red yeast rice (RYR), Hibiscus sabdariffa, and Gynostemma pentaphyllum, have been proven beneficial for lipid modification. Gynostemma pentaphyllum was initially eaten as a wild vegetable recorded in medicinal literature called "Materia Medica for Famines" (Jiuhuang Bencao) in China, an edible medicinal herb or a dietary supplement. Some commercial products from Gynostemma pentaphyllum, including tea and beverages, are available and are beneficial for hyperlipidemia. In addition, Gynostemma pentaphyllum was also used as additives in drinks, beer, bread, and noodles. Gynostemma pentaphyllum contains more than 20 chemical components such as gynostemma saponin, quercetin, and sitosterol. In 1976, Japanese expert Masahiro Nagai revealed that Gynostemma pentaphyllum contains dammarane saponins. And so far, more than 200 Gypenosides with well-defined structures have been found. Gypenoside is the major bioactive constituent of Gynostemma pentaphyllum. Modern pharmacological research supplies plentiful evidence of its anti-inflammatory, antihyperlipidemic ability and its regulatory role in liver function. Gypenoside can affect autophagy and lipid metabolism in cultured cells. Basic research found that Gypenoside consistently decreased TG, TC, and LDL-C more than simvastatin. The mechanisms of Gynostemma pentaphyllum in modulating lipids are related to inhibiting PCSK9, alleviating oxidative stress damage, and up-regulating expressions of Sirt1 and FOXO1 in macrophages with oxLDL.

\section{METHODS}

Search strategy: The Medline (via PubMed), the Cochrane Library, EMBASE, Web of Science, China National Knowledge Infrastructure (CNKI), Chinese Scientific Journal Database (VIP), SinoMed, and Wanfang Data will be searched in January 2022. We also explored the World Health Organization International Clinical Trials Registry Platform (https://www.who.int/ clinical-trials-registry-platform), ClinicalTrials.gov (www.clinicaltrials.gov/), 
and the Chinese Clinical Trial Registry (http://www.chictr.org.cn/index.aspx) for ongoing or unpublished trials to January 2022. Subject words or text words are determined based on published systematic reviews, clinical practice guidelines, ICD-10, ICD-11, MeSH terms, and Emtree.

Medline (via PubMed)

\#1 Gynostemma [mesh terms]

\#2 Gynostemma* or "Gynostemma

pentaphyllum*" or "pentaphyllum,

Gynostemma" or gypenoside or

gynoglycosides or Jiaogulan or "Jiao Gu

Lan" or Jiao-Gu-Lan [All Fields]

\#3 \#1 OR \#2

\#4 Dyslipidemias [mesh terms]

\#5 Triglycerides [mesh terms]

\#6 Cholesterol [mesh terms]

\#7 Lipoproteins [mesh terms]

\#8 Apolipoprotein A-I [mesh terms]

\#9 Apolipoproteins B [mesh terms]

\#10 Lipoprotein(a) [mesh terms]

\#11 Dyslipidemia* or Cholesterol or

Lipoprotein* or Lipid?emia* or Lipemia* or

"Lipase D Deficiency" or Hyperlipidemia or Dysbetalipoproteinemia[All Fields]

\#12 Dyslipoproteinemia* or Hyperlipid?

emia* or Hyperlipemia* or

Hyperprebetalipoproteinemia or

Hypercholesterol?emia* or

Hyperlipoprotein?emia* or

Hypertriglycerid?emia* or

Hyperalphalipoproteinaemia or "Broad

Beta Disease" or

Hypoalphalipoproteinemias or

Hypobetalipoproteinemias or

Hypolipoproteinemia* Or

Hypoprebetalipoproteinemia[All Fields]

\#13 "Total cholesterol" or TC or Triglyceride

or TG or "low-density lipoprotein

cholesterol" or LDL-C or "high-density

lipoprotein cholesterol" or HDL-C or

"Apolipoprotein A1" or "Apo A1" or "Apo

$A-1$ " or "Apolipoprotein B" or "Apo B" or

"Apo B100" or "Lipoprotein a" or "Lp a"[All

Fields]

\#14 or/4-13

In addition, the reference lists of all identified studies will also be searched to find any further relevant trials for inclusion.

Participant or population: We will include trials involve participants with dyslipidemia, of both genders and any age. Considering the diversity of dyslipidemia classifications and the changing diagnostic criteria, we will not limit specific diagnostic criteria.

Intervention: We will include all types of Gynostemma pentaphyllum, whether raw herb or extracts, used alone or combined with lipid-lowering agents.

Comparator: The control intervention will include no treatment, placebo, lipidlowering agents including but not limited to statins, Chinese herbal products that containing red yeast rice extracts. In addition, western herbs, which has been proved effective compared to placebo or lipid-lowering agents, will be included, too.

Study designs to be included: Randomized, parallel controlled trials will be included irrespective of blinding, publication status and language.

Eligibility criteria: We will include trials involve participants with dyslipidemia, of both genders and any age. We will include all types of Gynostemma pentaphyllum, whether raw herb or extracts, used alone or combined withconventional lipidlowering agents.The control intervention will include no treatment, placebo, lipidlowering agents including but not limited to statins, Chinese herbal products that containing red yeast rice extracts. In addition, western herbs, which has been proved effective compared to placebo or lipid-lowering agents, will be included.Randomized, parallel controlled trials will be included irrespective of blinding, publication status and language.

Information sources: The PubMed, the Cochrane Library, EMBASE, Web of Science, China National Knowledge Infrastructure (CNKI), Chinese Scientific Journal Database (VIP), SinoMed, and Wanfang Data will be searched in January 2022. We also explored the World Health Organization International Clinical Trials Registry Platform (https://www.who.int/ clinical-trials-registry-platform), ClinicalTrials.gov (www.clinicaltrials.gov/), and the Chinese Clinical Trial Registry (http://www.chictr.org.cn/index.aspx) for 
ongoing or unpublished trials to January 2022. Subject words or text words are determined based on published systematic reviews, clinical practice guidelines, ICD-10, ICD-11, MeSH terms, and Emtree. In addition, the reference lists of all identified studies will also be searched to find any relevant trials for inclusion.

Main outcome(s): The main outcome measures sought at the end of treatment and at maximal follow-up after completion of the treatment will be the number of people whose lipid level return to normal, and serum lipids levels, including but not limited to LDL-C, total cholesterol, triglyceride, and HDL-C.

Additional outcome(s): Additional outcomes will include cardiovascular events (both fatal and non-fatal events, including myocardial infarction, angina pectoris, stroke, peripheral arterial disease, sudden death), waist circumstances, body mass index, blood glucose, blood pressure, adverse events, and cost-effectiveness.

Data management: We will import all retrieved results into reference management software NoteExpress (Beijing Aegean Software company, Rev3.4.0.8878). Duplicate data from different databases will be identified first and screened by the software. Two reviewers (FFZ and FLP) will independently screen the titles and abstracts to select potential trials, and then review full texts for eligible trials according to the criteria described above. Any disagreements will be resolved through discussion or by consultation with a third review author (JPL). Data collection form will be designed using Microsoft Excel.

Quality assessment / Risk of bias analysis: The methodological quality of individual trials will be assessed by the Cochrane Risk of Bias tool 2.0. The tool includes the following five domains: randomization process, deviation from established interventions, outcome measures, missing outcome data, selective reporting of results, and overall bias. The included studies will be assessed as low risk of bias, some concern, or high risk of bias in each domain. The GRADE method will be used to rate evidential certainty in five domains, including risk of bias, directness, accuracy, consistency, and likelihood of publication bias.

Strategy of data synthesis: Data analyses will be performed by the Review Manager program (V.5.4.1 Copenhagen: The Nordic Cochrane Centre, The Cochrane Collaboration). Dichotomous data will be presented as relative risk (RR) and continuous data as mean difference (MD) for effect estimates, both with $95 \%$ confidence intervals (Cl). For dichotomous outcomes, studies with incomplete or missing data will be included in a sensitivity analysis by counting participants as treatment failure to explore the possible effect of loss to follow-up on the findings ('worst case' scenario). Meta-analysis will be performed if the study design, participants, interventions, control and outcome measures are sufficiently similar. 12 test will be used to assess heterogeneity. As recommended by the Cochrane Handbook 5.1.0, we define 12 as follows: (1) less than 50\%: represent mild heterogeneity; (2) 50\%-75\%: represent moderate heterogeneity; (3) more than 75\%: represent severe heterogeneity. A random-effect model will be used when the heterogeneity more than $75 \%$, otherwise the fixed effect model will be used.

Subgroup analysis: If sufficient data are available, the subgroup analysis will be carried out to reveal any effect that might explain any heterogeneity, including diagnostic criteria, control intervention, treatment duration.

Sensitivity analysis: We will ensure the stability of the comprehensive results by performing sensitivity analysis if necessary.

Language: No language limitations.

Country(ies) involved: This systematic review is being carried out in China.

Other relevant information: No. 
Keywords: Gynostemma pentaphyllum; lipid-lowering agents; Dyslipidemia; systematic review; meta-analysis; randomized controlled trials.

Dissemination plans: This systematic review results will be submitted for publication in peer review journal.

Contributions of each author:

Author 1 - Ning Dai - Writing - Original Draft, Formal Analysis. Ning Dai and FF Zhao are co-first authors.

Email: daining0719@bucm.edu.cn

Author 2 - Fangfang Zhao Conceptualization, Methodology, Formal Analysis, Data Curation, Writing - Review \& Editing.

Email: fionathio@126.com

Author 3 - Min Fang - Methodology, Software, Writing - Review \& Editing.

Email: fangmin353@163.com

Author 4 - Lingyao Kong - Methodology, Software, Writing - Review \& Editing.

Email: y153399885@163.com

Author 5 - Fenglan Pu - Methodology, Software, Formal Analysis, Data Curation, Writing - Original Draft.

Email: 18811361978@163.com

Author 6 - Jianping Liu - Conceptualization, Writing - Review \& Editing, Funding Acquisition.

Email: jianping_I@hotmail.com 